\title{
fVisiOn: glasses-free tabletop 3D display to provide virtual 3D media naturally alongside real media
}

\section{Shunsuke Yoshida}

Shunsuke Yoshida, "fVisiOn: glasses-free tabletop 3D display to provide virtual 3D media naturally alongside real media," Proc. SPIE 8384, ThreeDimensional Imaging, Visualization, and Display 2012, 838411 (15 May 2012); doi: 10.1117/12.920542

Event: SPIE Defense, Security, and Sensing, 2012, Baltimore, Maryland, United States 


\title{
fVisiOn: Glasses-free tabletop 3D display to provide virtual 3D media naturally alongside real media
}

\author{
Shunsuke Yoshida \\ National Institute of Information and Communications Technology (NICT), \\ 2-2-2 Hikaridai, Seika, Souraku, Kyoto 619-0288 Japan
}

\begin{abstract}
A novel glasses-free tabletop 3D display, named fVisiOn, floats virtual 3D objects on an empty, flat, tabletop surface and enables multiple viewers to observe raised 3D images from any angle at $360^{\circ}$.

Our glasses-free 3D image reproduction method employs a combination of an optical device and an array of projectors and produces continuous horizontal parallax in the direction of a circular path located above the table. The optical device shapes a hollow cone and works as an anisotropic diffuser. The circularly arranged projectors cast numerous rays into the optical device. Each ray represents a particular ray that passes a corresponding point on a virtual object's surface and orients toward a viewing area around the table. At any viewpoint on the ring-shaped viewing area, both eyes collect fractional images from different projectors, and all the viewers around the table can perceive the scene as 3D from their perspectives because the images include binocular disparity.

The entire principle is installed beneath the table, so the tabletop area remains clear. No ordinary tabletop activities are disturbed. Many people can naturally share the 3D images displayed together with real objects on the table.

In our latest prototype, we employed a handmade optical device and an array of over 100 tiny projectors. This configuration reproduces static and animated 3D scenes for a $130^{\circ}$ viewing area and allows 5 -cm-tall virtual characters to play soccer and dance on the table.
\end{abstract}

Keywords: Glasses-free 3D display, auto-stereoscopic 3D display, horizontal parallax, ring-shaped viewing area, tabletop interaction, virtual object, mixed reality

\section{INTRODUCTION}

We propose fVisiOn, a novel, glasses-free tabletop 3D display that augments ordinary tabletop communications by providing virtual 3D media naturally alongside real media. The display floats virtual 3D objects on an empty, flat tabletop surface and enables multiple viewers to observe each raised 3D image, like a centerpiece, from any angle at $360^{\circ}$. It provides a natural mixed-reality environment for multiple people around a table. Fig. 1 illustrates a tabletop interaction scenario and shows a developed mixed-reality environment on fVisiOn.

The display's viewing style is specifically designed for tabletop tasks, assuming a seated condition. Its principle of 3D image reproduction employs a combination of an optical device and an array of projectors that are installed beneath the table to keep the tabletop area clear. The projectors are arranged in a circular manner and produce numerous rays that travel in any direction. The optical device catches the rays and appropriately controls the traveling orientation to optimize viewing for the seated users. The directional rays fill a certain volume on the table and reproduce a light field to represent any virtual 3D object. This principle forms a ring-shaped viewing area that produces a parallax of depth perception in the direction of a horizontal circular path located above the table.

In this paper, we first describe the design concept of fVisiOn and discuss its novel, glasses-free 3D display principle based on light-field reproduction. Then we explain an implementation method and show some final 3D images reproduced by the prototype.

E-mail: shun@nict.go.jp, Telephone: +81-774-95-1453, Web: http://urv.nict.go.jp/member/shun/

Three-Dimensional Imaging, Visualization, and Display 2012, edited by Bahram Javidi, Jung-Young Son, Proceedings of SPIE Vol. 8384, 838411 - (c) 2012 SPIE

CCC code: $0277-786 X / 12 / \$ 18 \cdot$ doi: $10.1117 / 12.920542$

Proc. of SPIE Vol. $8384838411-1$ 

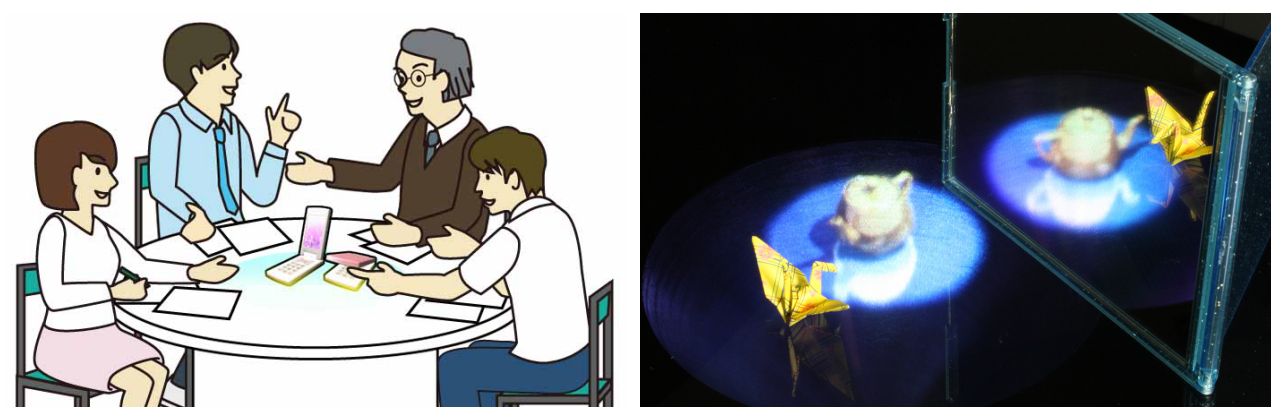

Figure 1. Conceptual illustration of a tabletop interaction scenario (left) and a mixed-reality environment on our proposed glasses-free tabletop 3D display: fVisiOn (right). A virtual "utha teapot" is displayed alongside a real origami crane. A mirror placed on the table's right side reflects the virtual and real $3 \mathrm{D}$ objects as if the scene were actually there.

\section{DESIGN CONCEPT}

\subsection{Tabletop Communications}

The surface area of a table, a tabletop, is a shared space for collaborative work that is useful for a variety of tasks. Many documents, materials, mock-ups, objects, and information can be shared and exchanged on tabletops to perform tasks involving multiple people seated around the table. One target scenario of fVisiOn is to support such collaborative work on tabletops in mixed-reality environments, where virtual 3D media can be naturally provided alongside real media.

To support such communications on tabletops, a display method that provides 3D media on the tabletop needs to satisfy the following requirements:

(1) The 3D images should be observed by each viewer with the correct perspective;

(2) Ordinary tabletop interaction activities should not be inhibited;

(3) The number of viewers should not be limited;

(4) Ideally, to facilitate natural communication, no special 3D glasses or wearable tracking systems should be required.

\subsection{Related Works on 3D Displays}

There have been many research efforts to develop systems based on tabletop interaction, and some have focused on providing 3D visual information on table surfaces. ${ }^{1,2}$ These displays satisfy conditions (1) and (2), but they require special 3D glasses ${ }^{1}$ and head tracking. ${ }^{1,2}$ Wearing glasses sometimes causes discomfort, and the latter device usually limits the number of tabletop users, thus they do not simultaneously satisfy conditions (3) and (4).

In recent years, many auto-stereoscopic 3D displays have been proposed, ${ }^{3,4}$ and several related works envision similar design concepts, although each of these displays only satisfies some of the above conditions.

Full parallax 3D displays based on an integral imaging technique ${ }^{5}$ can be laid on a table, and they satisfy conditions (1), (2), and (4). However, the typical viewing area of the display is narrow. This method is principally appropriate for expressing depth information, and the best observation direction is almost directly in front of the display (from the top for tabletop use). ${ }^{6}$ Viewing is not suitable from a slanted perspective above the table, such as that of the people seated around it. Consequently, a method was proposed for optimizing the table's viewing area. ${ }^{7}$ This viewing area occupies an oblique position above the table. Nevertheless, this method only provides an auto-stereoscopic effect within a small space in a particular direction, so the method does not satisfy conditions (1) and (3). 

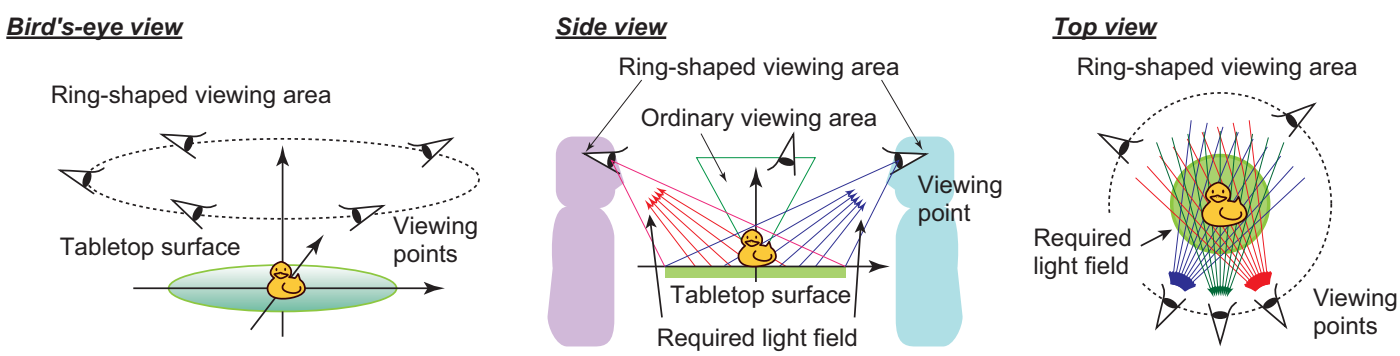

Figure 2. Required light field and ring-shaped viewing area for tabletop 3D display.

Volumetric (volume-swept) displays ${ }^{8}$ and recently expanded approaches based on light-field reproduction ${ }^{9-11}$ are other candidates. Such glasses-free 3D display provides binocular and motion parallax along the circular direction in the horizontal plane: the so-called horizontal-parallax-only (HPO) 3D display. Therefore, it can generate 3D images of $360^{\circ}$ and satisfies conditions (1), (3), and (4). However, it requires a number of mechanical moving components and a big glass case that involves the components inside and invades the tabletop space. Thus there is a critical limitation toward satisfying condition (2).

\subsection{Proposed Tabletop 3D Display}

The 3D image-reproduction principle of fVisiOn is designed to meet all four conditions.

The complete mechanism is installed underneath the table to satisfy condition (2). It does not occupy the tabletop area and naturally supplies virtual $3 \mathrm{D}$ objects for ordinary tabletop tasks. The display forms a $360^{\circ}$ circular viewing area around the table and provides horizontal parallax without 3D glasses or head tracking in the horizontal plane to satisfy conditions (3) and (4). Therefore, any number of viewers seated around the table, as in condition (1), can observe the 3D images with a correct perspective from any direction.

\section{LIGHT-FIELD REPRODUCTION FOR TABLETOP 3D DISPLAY}

\subsection{Principle of Light-field Reproduction}

In a real setting, objects are illuminated by light sources, and any diffusion-reflective surface of the objects reflects the light in all directions with different luminosity and wavelength properties. It is generally accepted that a virtual point light source exists at every point on the surface. 3D displays based on light-field reproduction simulate this physical lighting condition by employing numerous directional rays using an appropriate method. In geometric optics, the radiation of light is briefly represented by rays; therefore, the point light source radiates an uncountable number of rays in all directions. The directional rays fill a certain volume and form a "light field." Any intersection of the rays works as a virtual point light source. If a sufficient number of directional rays exist in the light field, a virtual 3D scene can be reproduced.

Ordinary flat panel 3D displays are generally designed to be viewed perpendicularly. Viewers can observe accurately 3D images only in this narrow area, which is called the viewing area. In contrast, when assuming a tabletop interaction scenario, the 3D images on the table are observed chiefly from a slanted direction above the table. This means that the viewing area should be optimized for seating around the table, requiring a sufficient light field for each direction and a certain viewing height. Fig. 2 illustrates the conditions of the required light field and the appropriate ring-shaped viewing area for a tabletop 3D display.

\subsection{Reproduction of Appropriate Light Field}

fVisiOn reproduces the necessary conditions seen in Fig. 2 by employing an optical device and many projectors. ${ }^{12}$

The optical device is a revolving body and uses an anisotropic radiation character for incoming rays; it diffuses in the direction of the edge line of the shape and passes straight in the direction of the circumference. It works as a kind of rear-projection screen, so below we simply call it a "screen." The combination of the screen and the projector array can provide horizontal parallax in a circular direction (Fig. 3). 

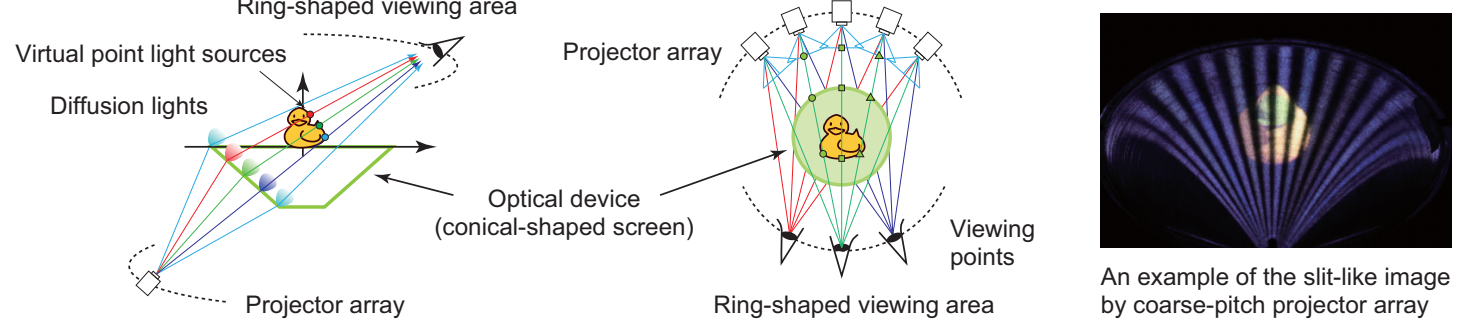

Figure 3. Light-field reproduction principle of fVisiOn.

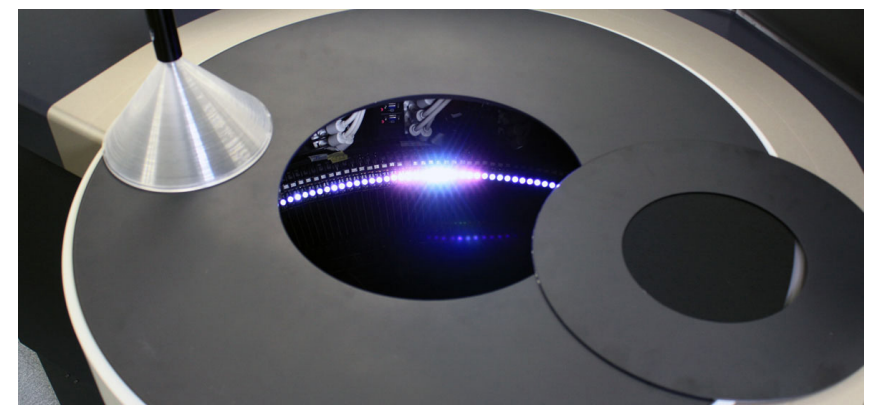

Figure 4. Screen and projector array. Many dots seen from table's opened hatch are the micro-projector's lights. Cone on left side is the conical screen placed temporarily upside-down on the table.

On a vertical plane, a ray enters the screen and extends at a certain angle. Part of the fan-like diffused light from the ray is caught by the viewer's eye (left-side, Fig. 3).

In contrast, on a horizontal plane, the orientation of the rays produced from a series of projection centers is not changed after they pass through the screen (middle, Fig. 3).

The ring-shaped viewing area is defined as continuous viewing points. At any eye position on the ring-shaped viewing area, the eye observes certain slit-like parts in each projector's rays. Therefore, the retina collects fractional slit-like images from different projectors and form an appropriate image for the perspective at each viewing point (right, Fig. 3). Another eye located at a separate position sees a different grouping of rays even from the same projectors. Since a pair of these individual-perspective images contains parallax, the viewers perceive 3D images on the tabletop due to binocular disparity without 3D glasses.

\section{PROTOTYPE IMPLEMENTATION}

\subsection{Optical Device as Screen}

The principle requires the shape of a revolving body and an anisotropic scattering characteristic for incoming rays. We made our prototype screen in the following steps.

First, the core of the screen is made from acrylic resin and forms a hollow, 12-cm deep cone with a 20-cm diameter. Viewers effectively look down the side surface of the hollow cone. To apply the anisotropic optical function required for this principle, a filament-like lens is wound on the surface of its side. We employed a nylon fishing line as the filament-like lens. The line was wound and bonded on the cone's surface using an ultraviolet-curing adhesive.

In practice, our prototype screen showed an angular characteristic of around $0.2^{\circ}$ in the circumference direction of the cone and $60^{\circ}$ in the vertical direction. ${ }^{13}$

The screen is installed just beneath the tabletop surface, which is made from a 3-mm-thick black transparent acrylic plate and leveled $70 \mathrm{~cm}$ from the floor, which is the height of an ordinary table (Fig. 4.) 


\subsection{Projector Array}

As sources to generate numerous directional rays, many projectors must be arranged in a circle and as close together as possible. At present, LCD projectors for mobile use (VGA, 8-lm) are relatively effective in this regard due to their image quality, cost performance, and small projection unit size. The one we used is $15-\mathrm{mm}$ thick.

The width of each slit-like fractional image on the screen is related to the scattering characteristics in the circumference direction; the angular pitches of the projectors should equal the scattering characteristics. Here, the projector interval is physically restricted, and a 15- $\mathrm{mm}$ arc corresponds to an angular pitch of $0.2^{\circ}$ at $4.3 \mathrm{~m}$ of projection distance, but this is too long. In the prototype, we chose $87 \mathrm{~cm}$, even though this corresponds to around $1^{\circ}$ of the angular pitch. To adjust the mismatch between it and the scattering characteristics, an isotropic diffuser of $0.5^{\circ}$ was applied to the screen. Since the diffuser blurs the final images, the angular pitch must be shortened to generate sharper results.

Our latest prototype includes over 100 precisely aligned tiny projectors (Fig. 4). To avoid the troublesome mechanical alignment of so many projectors, their optical axes are roughly concentrated at a certain point physically, and then precise software correction is applied. The correction parameters are assigned by measurements of the known patterns projected on a predefined plane.

\subsection{Controllers}

For supplying hundreds of source images in a smaller number of controllers, we employ a middle-end computer equipped with four graphic cards that have two output channels. The computers outputs images of $1920 \times 480$ pixels for each channel. They are then separated into three VGA images before being supplied to our VGA projectors. Thus, in this configuration, one computer simultaneously manages 24 projectors.

Our prototype employs five computers for supplying the source images and one additional computer for managing the others. The control of the frame synchronization, such as seen in sequential playing for animation, is simply performed by broadcasting special packets from the managing computer by Ethernet.

\section{CREATION OF VIRTUAL 3D SCENE}

\subsection{Generation of Source Images}

The projector array projects appropriate individual images onto the side surface of the conical screen from different directions. Each pixel of the images forms numerous directional rays passing through each projection center and the pixel's projected point. A source image for each projector is computed based on a ray-tracing algorithm. ${ }^{13}$

The traced ray starts from any eye position on the ring-shaped viewing area, and we obtain intersecting points at the imaginary object's surface and the screen surface. The directional ray produced from a certain intersecting point is emitted by the color property of the object's intersecting point. Therefore, the intersecting point at the screen must be assigned the same color property along the ray. Then, an image to be projected is determined by the relationship between the projected surface of the screen and the projection center of a projector (Fig. 3).

The ring-shaped viewing area is defined by considering a tabletop interaction scenario, such as hand-manipulation of virtual 3D objects; its radius is $50 \mathrm{~cm}$ and its height is $34 \mathrm{~cm}$ from the tabletop. In the scenarios, participants are usually seated, and this situation gently leads their eyes to the expected viewing area. The correct reproduced 3D images are only observed at the viewpoints on the assumed ring-shaped viewing area. The vertical diffusion due to the anisotropic optical function of the screen guarantees observation from a slightly different position from the viewing area, but the perceived 3D images are deformed based on the above principle of source image generation.

The source images are supplied by VGA, $640 \times 480$ pixels, but a valid projection area is currently limited to around $100 \times 100$ pixels. Since our research remains in the prototype stage, we employed uncustomized commercial projectors for proving our 3D image reproduction principle. The current optics derived from a preinstalled lens system of the projector is wider than the ideal configuration. Optimization of the lens system is required for improving the quality of the final reproduced 3D images. 

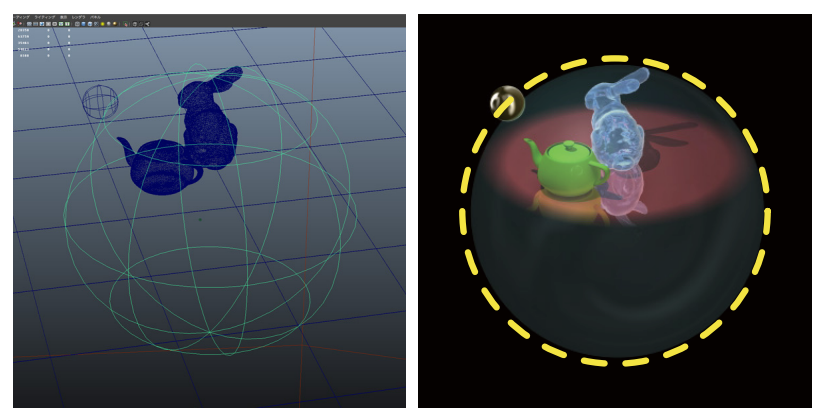

Figure 5. Creation process of virtual 3D scene using proposed guidance information: "safe volume." The wire-framed spherical pale gray area is the safe volume displayed on commercial modeling software. A dotted yellow circle was post-inserted for easy reading.

\subsection{Creation of Scenes by a Guide}

In the target scenarios of such a tabletop 3D display, the reproduced 3D scene is expected to be seen from any viewing direction around the table. For maintaining the perfect appearance of the scene for $360^{\circ}$ observation, content creators and designers of virtual 3D scenes should ideally well understand its display mechanism and restrictions, but the emerging technologies of $3 \mathrm{D}$ displays are sometimes difficult for non-specialists who are concentrating on creative works rather than learning 3D display technologies. For supporting content productions, we analyzed restrictions in 3D scene creations related to tabletop 3D display configurations and proposed a method for easily creating appropriate 3D scenes. ${ }^{14}$

"Safe volume" is our name for the area available for placing any virtual object and for omni-directional viewing. It is derived from the geometrical configuration; i.e., as a minimum, any objects situated inside the volume are guaranteed to be seen from any viewpoint on the ring-shaped viewing area.

The safe volume is defined as a body of an intersection formed by the rotary sweeping of a triangle, which has a vertex located at any viewpoint on the ring-shaped viewing area, and two edges that connect the vertex and the top or the bottom of the side surface of the conical screen (side-view, Fig. 3). A simplified primitive shape of a sphere inscribed in the rotary sweeping body is also reasonable to be employed as the safe volume.

In our content production approach, scene creators merely load the shape data of the precomputed safe volume or simply make a primitive shape of a sphere on their favorite modeling software. Then they can freely build their own world inside the safe volume (Fig. 5). While constructing virtual 3D scenes, there is no limitation on the camera work of the modeling software except small suggestions. One is to render the scene from the ringshaped viewing area while aiming at the center of the tabletop surface for previews. For floating 3D scenes from the table, only the upper semi-sphere should be employed. Also note that the center of the tabletop surface does not usually equal the center of the safe volume.

In the configuration of our latest prototype, the spherical safe volume is computed as a space of around $10 \mathrm{~cm}$ in diameter, so the virtual objects are raised $5 \mathrm{~cm}$ from the table's surface. The center of the safe volume derived from the relationship between the screen and the viewing area was slightly lower than the tabletop surface level.

\section{REPRODUCED 3D IMAGES}

We created several virtual 3D scenes and reproduced them on our prototyped glasses-free tabletop 3D display.

Fig. 6 shows several results of produced static 3D images photographed from a certain viewpoint on the ring-shaped viewing area. Each virtual 3D object is displayed at a volume of around $10 \mathrm{~cm}$ in diameter at the center of the tabletop and raised around $5 \mathrm{~cm}$ from the tabletop surface.

Fig. 7 is another example. The scene, which contains an imaginary rabbit and a real origami crane, was taken from different angles at three separated viewpoints on the ring-shaped viewing area. From the figure, we can confirm that the reproduced 3D images provide parallax without special glasses. Note that reflection and 

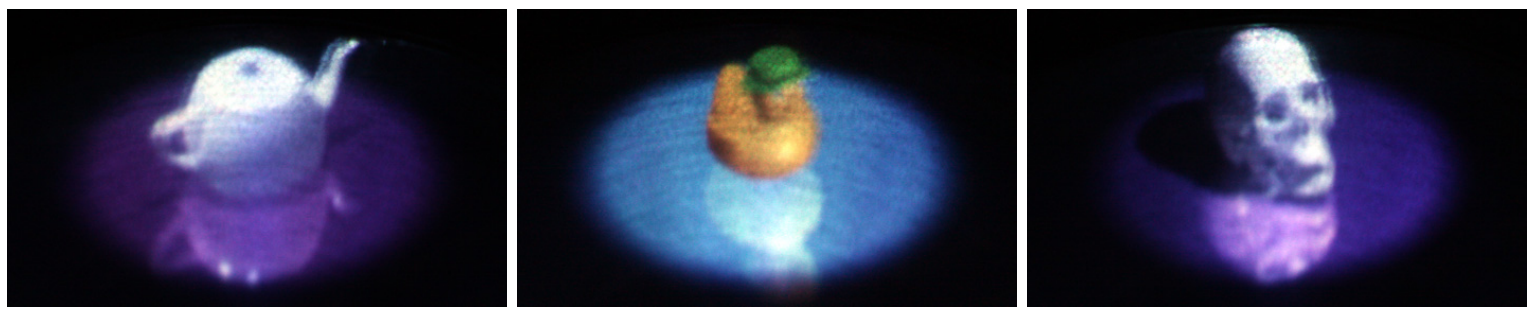

Figure 6. Snapshots of 3D images reproduced by proposed tabletop 3D display. Displayed imaginary scenes include a teapot, a toy duck, and a skull.
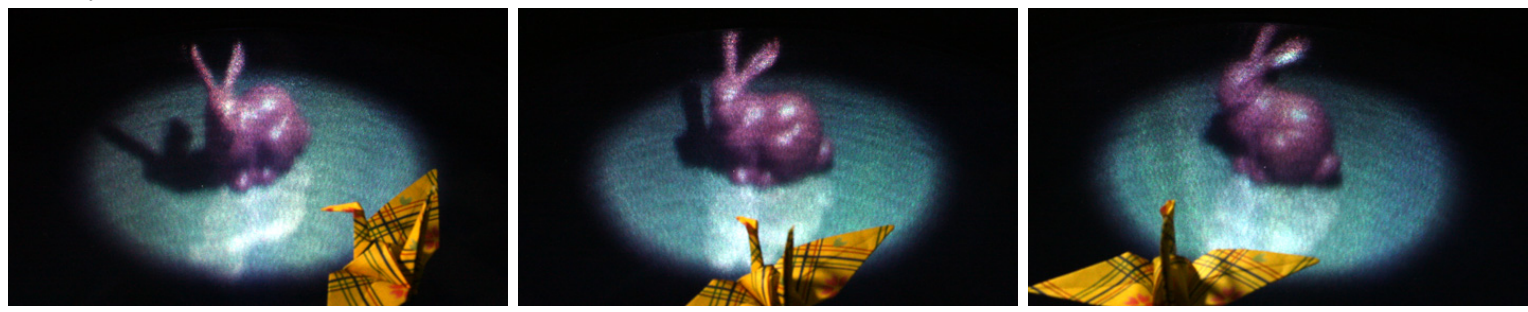

Figure 7. Snapshots taken from different angles. Perspectives of CG Stanford Bunny with a real origami crane are different in each observation.

the shadow cast on the imaginary green circular plate on the tabletop surface are also reproduced appropriately due to rendering the source image based on the ray-tracing algorithm.

Fig. 8 shows sequential images of an animated 3D scene of a soccer corner kick from a unique viewpoint. In the current scene creation process, a scene built on commercial modeling software is rendered by every frame of the sequential animation in advance. When an animator creates animation for the 3D scene, a custommade rendering script automatically generates the source images for each frame. All frames are then played continuously on each computer with synchronization of $30 \mathrm{~Hz}$. The creator of this animation did not have any knowledge of our 3D display except for the safe volume sphere. By employing safe volume, 3D scene creators can simply build their own worlds without complicated knowledge of emerging 3D displays. As a result, we confirmed that 5 -cm-tall players can run and kick the ball as if they were playing the game on the table under a spotlight. Other supplemental animations that include a miniature concert scene of a virtual female singer are demonstrated on the Internet. These extra video contents are available at http://youtu.be/u1E_LgLaiRE.

\section{CONCLUSION}

This paper described a design concept and an implementation method for a novel, glasses-free tabletop 3D display. Full-color, 5-cm tall 3D characters are animated on a flat tabletop surface, and several viewers can share and enjoy the 3D images from appropriate perspectives for each position.

fVisiOn has high affinity with ordinary collaborative activities on tabletops and provides natural mixed-reality environments. It would be appropriate for group discussions and teleconferences using digital documents and virtual mock-ups around the table.

Another example is supporting operations based on such geographic information on maps as urban planning, traffic control, and disaster prevention because this display is designed for a seated condition that usually brings
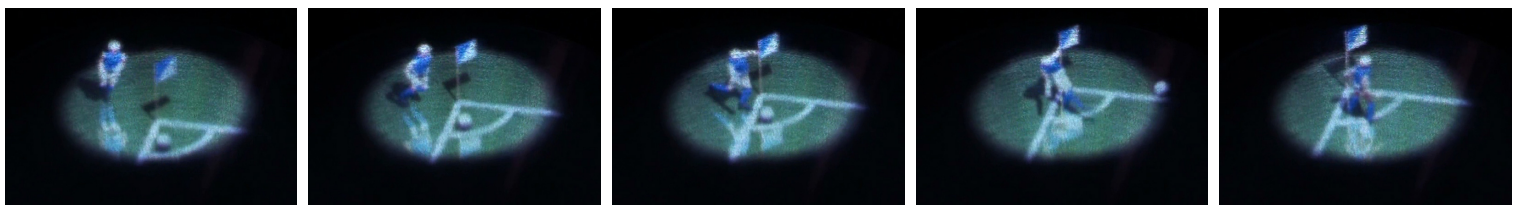

Figure 8. Sequential snapshots of reproduced animated 3D scene shot from a unique viewpoint. 
an oblique bird's eye view. The system also promises to be suitable for medical applications, i.e., obtaining informed consent and simulating operations; glasses-free 3D display might not disturb natural conversations among doctors and patients.

Since the system has no high-speed controlled components or moving devices, it is more appropriate for displaying full-color and real-time animation and for enlarging the display area. As one ultimate goal, this technique has the potential to construct a 3D arena, since its viewing format resembles a sports stadium. Our current implemented system covers approximately $130^{\circ}$ of the observation area, although this is around one-third of the ideal due to the limited number of projectors. In the future, a full $360^{\circ}$-view tabletop $3 \mathrm{D}$ display will be developed.

The reproduced 3D images are somewhat blurred and unfocused. Refining the 3D image quality by improving hardware equipment and configurations as well as software algorithms will be future work. For example, when the display's resolution is limited as implemented in this configuration, characters of high-contrast color and easyto-understand appearance might be better. Future work will also study a creation guide of virtual characters based on the resolution of the 3D display. The current reproduced 3D objects in the demonstrated scenes are all imaginary 3D models. Capturing and reproducing real 3D scenery is additional future work.

\section{ACKNOWLEDGMENTS}

Part of this research was supported by the Japan Science and Technology Agency (JST), Core Research for Evolutional Science \& Technology (CREST).

\section{REFERENCES}

[1] Kitamura, Y., Konishi, T., Yamamoto, S., and Kishino, F., "Interactive stereoscopic display for three or more users," in [Proceedings of the 28th annual conference on Computer graphics and interactive techniques], SIGGRAPH '01, 231-240, ACM, New York, NY, USA (2001).

[2] Ye, G., State, A., and Fuchs, H., "A practical multi-viewer tabletop autostereoscopic display," in [Mixed and Augmented Reality (ISMAR), 2010 9th IEEE International Symposium on], 147 -156 (2010).

[3] Holografika, [HoloVisio]. http://www.holografika.com/.

[4] Iwasawa, S., Kawakita, M., Yano, S., Sakai, M., Haino, Y., Sato, M., and Inoue, N., "A 200-inch 3D-glassesfree high-definition projection display," in [2011 SMPTE Technical Conference \& Exhibition], 2011(1), 1-3 (2011).

[5] Lippmann, M. G., "Épreuves réversibles donnant la sensation du relief," J. Phys. Theor. Appl. 7, 821-825 (1908).

[6] Arai, J., Okui, M., Kobayashi, M., and Okano, F., "Geometrical effects of positional errors in integral photography," J. Opt. Soc. Am. A 21(6), 951-958 (2004).

[7] Fukushima, R., Taira, K., Saishu, T., and Hirayama, Y., "Novel viewing zone control method for computergenerated integral 3-D imaging," Stereoscopic Displays and Virtual Reality Systems XI 5291(1), 81-92, SPIE (2004).

[8] Favalora, G. E., "Volumetric 3D displays and application infrastructure," Computer 38, 37-44 (August 2005).

[9] Jones, A., McDowall, I., Yamada, H., Bolas, M., and Debevec, P., "Rendering for an interactive $360^{\circ}$ light field display," in [ACM SIGGRAPH 2007 papers], SIGGRAPH '07, ACM, New York, NY, USA (2007).

[10] Ito, K., Kikuchi, H., Sakurai, H., Kobayashi, I., Yasunaga, H., Mori, H., Tokuyama, K., Ishikawa, H., Hayasaka, K., and Yanagisawa, H., "360-degree autostereoscopic display," in [ACM SIGGRAPH 2010 Emerging Technologies], SIGGRAPH '10, 1:1-1:1, ACM, New York, NY, USA (2010).

[11] Endo, T., Kajiki, Y., Honda, T., and Sato, M., "Cylindrical 3-D video display observable from all directions," in [Computer Graphics and Applications, 2000. Proceedings. The Eighth Pacific Conference on], $300-306$ (2000).

[12] Yoshida, S., Yano, S., and Ando, H., "Prototyping of glasses-free table-style 3D display for tabletop tasks," in [SID International Symposium], 211-214 (2010). 
[13] Yoshida, S., Kawakita, M., and Ando, H., "Light-field generation by several screen types for glasses-free tabletop 3D display," in [3DTV Conference: The True Vision - Capture, Transmission and Display of 3D Video (3DTV-CON), 2011], 1 -4 (may 2011).

[14] Yoshida, S., "Parameterization of sharable display area to reproduce appropriate glasses-free tabletop 3-D images," in [IEEE Virtual Reality 2012], 131-132 (march 2012). 